\title{
Try before you buy - Akzeptanz von Virtual-Reality-Anwendungen zur Leistungsbeurteilung von Erfahrungsgütern
}

\author{
Kai Israel • Christopher Zerres • Dieter K. Tscheulin
}

Eingegangen: 16. Juli 2021 / Angenommen: 17. November 2021 / Online publiziert: 9. Dezember 2021 (C) Der/die Autor(en) 2021

Zusammenfassung Virtual-Reality-Anwendungen ermöglichen es Anbietern von Erfahrungsgütern durch innovative Produktpräsentationen die inhärenten Informationsasymmetrien zu reduzieren. Dadurch kann den potenziellen Kunden eine effiziente Leistungsbeurteilung ermöglicht und das Risiko einer informationsbedingten Fehlentscheidung minimiert werden. Die vorliegende Studie fokussiert sich auf die Identifikation wichtiger Determinanten, die die Nutzungsintention von Virtual-Reality-Anwendungen zur Leistungsbeurteilung von Erfahrungsgütern beeinflussen. Um das Akzeptanzverhalten von Nutzern gegenüber dieser neuartigen Technologie zu erforschen, wurde ein erweitertes Technologieakzeptanzmodell eingesetzt. Als Untersuchungsobjekt wurde eigens für die Studie eine Virtual-Reality-Anwendung entwickelt, die es den Nutzern ermöglichte, eigenständig ein virtuelles Erfahrungsgut zu erkunden. Insgesamt nahmen 569 Probanden an der Datenerhebung teil. Für die Berechnung des Strukturgleichungsmodells und die Hypothesenüberprüfung wurde eine Partial-Least-Squares-Analyse eingesetzt. Wie die Studienergebnisse verdeutlichen, führt das immersive Produkterlebnis zu einer effizienteren Informationsbeschaffung. Speziell der wahrgenommene Nutzen einer Virtual-Reality-Anwendung ist ein zentraler Prädiktor, der sowohl auf die Nutzungseinstellung als auch auf die Nutzungsintention einen starken positiven Einfluss ausübt.

Schlüsselwörter Virtual-Reality · Technologieakzeptanz · Erfahrungsgüter · Produktpräsentation

\footnotetext{
Kai Israel · Christopher Zerres $(\bowtie)$

Fakultät Medien, Hochschule Offenburg, Badstraße 24, 77652 Offenburg, Deutschland

E-Mail: christopher.zerres@hs-offenburg.de

Dieter K. Tscheulin

Fakultät für Betriebswirtschaftslehre, Universität Freiburg, Rempartstraße 16, 79098 Freiburg,

Deutschland
} 


\title{
Try before you buy-Acceptance of Virtual Reality Applications for the Evaluation of Experience Goods
}

\begin{abstract}
Virtual reality applications enable providers of experience goods to reduce the inherent information asymmetries through innovative product presentations. Thereby, potential customers can efficiently assess the product performance and minimize the risk of an information-related wrong decision. This study focuses on identifying important determinants that influence the intention to use virtual reality applications for performance evaluation of experience goods. To explore users' acceptance behavior towards this new technology, an extended technology acceptance model was employed. A virtual reality application that allowed users to independently explore a virtual environment was developed for the study. A total of 569 individuals participated in the study. Partial least squares analysis was used to calculate the structural equation model and for the hypothesis testing. As the study results illustrate, the immersive product experience leads to more efficient information gathering. Specifically, the perceived usefulness of a virtual reality application is a key predictor that has a strong positive influence on the variables attitudes towards using the technology and intention to use it.
\end{abstract}

Keywords Virtual reality · Technology acceptance $\cdot$ Experience goods · Product presentation

\section{Einführung}

Die zunehmende Digitalisierung und die fortwährende Weiterentwicklung webbasierter Technologien eröffnen für nahezu alle Branchen neue Möglichkeiten, um potenziellen Kunden relevante Produktinformationen zur Verfügung zu stellen. Insbesondere bei Erfahrungsgütern besteht für den Konsumenten keine Möglichkeit, das Produkt vor der Nutzung zu testen und dessen Qualität zu beurteilen (Sjurts 2011). Demzufolge kann der Konsument seine Kaufentscheidung hauptsächlich auf Basis der vorab zur Verfügung stehenden (Produkt-)Informationen treffen. Daher müssen dem Konsumenten relevante Daten in der Informationsbeschaffungsphase bereitgestellt werden, die die Produkteigenschaften möglichst exakt beschreiben (Häubl und Trifts 2000). Neben browserbasierten Informationsquellen eröffnen Virtual-RealityAnwendungen (VR-Anwendungen) neue Formen der Produktpräsentation (Hollebeek et al. 2020). Virtual-Reality wird definiert als ,, a completely immersive virtual and aural world that a user experiences, usually through a head-mounted display" (PWC 2018, S. 2), „that simulates a user's physical presence and environment in a way that allows the user to interact with it" (Isaac 2016). Die außergewöhnlichen Visualisierungsmöglichkeiten von VR-Anwendungen sind dazu in der Lage, dem potenziellen Kunden das Gefühl zu vermitteln, sich unmittelbar vor Ort zu befinden, wodurch insbesondere Erfahrungsgüter bereits im Vorfeld des Kaufs besser beurteilt werden können. Diesen Vorteil nutzen vermehrt Unternehmen wie beispielsweise AIDA Cruises, Kempinski oder der Europa-Park, um ihre Produkte etwa in Reisebüros erfahrbar zu machen (diginetmedia 2021). Die zunehmende Verbreitung von 
VR-Systemen wird auch durch die steigenden Absatzzahlen deutlich. Bereits heute befinden sich schätzungsweise 19Mio. VR-Systeme weltweit im Einsatz (Omdia 2020).

Im Rahmen des vorliegenden Beitrages wird auf Basis des Technologieakzeptanzmodells (TAM) untersucht, welche Faktoren die Nutzungseinstellung sowie Nutzungsintention von VR-Anwendungen beeinflussen. Daneben wird die Rolle der Telepräsenz als zentrales Merkmal von VR-Anwendungen und deren Einfluss auf die Technologieakzeptanz thematisiert.

\section{Konzeptionelle Grundlagen und Hypothesenherleitung}

\subsection{Technologieakzeptanzmodell}

Um die Akzeptanz eines Nutzers gegenüber neuartigen Technologien zu ermitteln, hat sich das TAM etabliert (Manis und Choi 2019; Legris et al. 2003; King und He 2006). Basierend auf der Theorie des überlegten Handelns, die die Beziehung zwischen Einstellung und Verhaltensabsicht erklärt (Fishbein und Ajzen 1975), entwickelte Davis (1989) ein Modell, um die Nutzerakzeptanz von Informationssystemen zu beschreiben. Die Nutzungsintention, definiert als „one's intention to perform a specified behavior" (Davis et al. 1989, S. 984), ist dabei maßgeblich von der individuellen Nutzungseinstellung (,,an individual's positive or negative feelings about performing the target behavior") (Davis et al. 1989, S. 984) gegenüber Informationstechnologien abhängig. Diese werden primär von den kognitiven Faktoren des wahrgenommenen Nutzens sowie der einfachen Bedienbarkeit beeinflusst (Davis et al. 1989). Den wahrgenommenen Nutzen beschreibt Davis als ,the degree to which a person believes that using a particular system would enhance his or her job performance“, wohingegen die einfache Bedienbarkeit als "the degree to which a person believes that using a particular system would be free of effort“ definiert wird (Davis 1989, S. 320).

Insbesondere die inhärente Informationsasymmetrie, die bei Erfahrungsgütern vorliegt, muss sukzessive minimiert werden, um potenzielle Kunden vom Angebot zu überzeugen. Gerade durch die zum Teil erheblichen finanziellen Aufwendungen für den potenziellen Konsumenten (z. b. bei Reiseangeboten), ist ein höheres Maß an Such- beziehungsweise Planungsaufwand im Kaufentscheidungsprozess notwendig, um eine fundierte Entscheidung treffen zu können (Xiang et al. 2015). Nahezu alle Kaufentscheidungen werden in diesem Zusammenhang auf Basis von Unsicherheiten gefällt, da sowohl die unmittelbaren Ergebnisse als auch mittel- bis langfristige Konsequenzen, die sich durch den Kauf ergeben, im Vorfeld nicht bekannt sind (Taylor 1974). Dementsprechend besteht für den Verbraucher immer das Risiko, eine informationsbedingte Fehlentscheidung zu treffen. Um dieses Risiko zu minimieren, wird der Verbraucher nach Informationen suchen, die für den Kauf von Relevanz sind (Flanagin et al. 2014; Shim und Lee 2011; Taylor 1974). Die Informationssuche wird auf Basis des Kosten-Nutzen-Prinzips durchgeführt, wobei es das Anliegen des Verbrauchers ist diese möglichst effizient durchzuführen. Kosten beziehen sich in diesem Zusammenhang auf die beanspruchte Zeit, die benötigten 
finanziellen Mittel sowie auf geistige Anstrengungen, die die Verbraucher für die Informationsrecherche aufwenden müssen. Der Nutzen entsteht durch die Risikoreduktion, welche von den Verbrauchern durch die Informationssuche wahrgenommen wird (Wu et al. 2008; Dowling und Staelin 1994). Die Verbraucher werden daher die Informationssuche solange fortsetzen, bis der Nutzen in einem positiven Verhältnis zu den aufgewendeten Kosten steht oder sich keine weiteren Informationen mehr beschaffen lassen und daher keine Entscheidung getroffen wird.

Ein geeignetes Instrument, um die Informationsasymmetrie zu reduzieren, können VR-Anwendungen sein. Insbesondere durch eine realistische Darstellung des Produktes und der intuitiven Interaktion wird dem Nutzer im Informationsbeschaffungsprozess das Gefühl vermittelt, sich unmittelbar vor Ort zu befinden (Buhalis und Law 2008). Auf Basis der zur Verfügung gestellten Informationen können die Nutzer das Leistungsangebot besser beurteilen, wodurch die Wahrscheinlichkeit einer Fehlentscheidung reduziert wird (Tseng und Wang 2016; Shim und Lee 2011). Vor allem im Online-Bereich sind die bereitgestellten Produktinformationen von elementarer Bedeutung, da die Kunden nur anhand dieser Informationen das Leistungsangebot beurteilen können und daher die Kaufbereitschaft von den bereitgestellten Produktinformationen beeinflusst wird (Li et al. 2001; Kaplanidou und Vogt 2006). Speziell interaktive Produktdarstellungen führen in diesem Zusammenhang zu einer höheren Konsumentenzufriedenheit, wodurch die Nutzungseinstellung gesteigert werden kann (Kim und Forsythe 2008; Lee et al. 2006). Neben der Nutzungseinstellung hat laut Davis (1989) der wahrgenommene Nutzen ebenfalls einen direkten Einfluss auf die Nutzungsintention. Dieser Umstand begründet sich in der Annahme, dass ein Informationssystem, welches dem Nutzer Effizienzvorteile bietet, auch zukünftig vom Nutzer verwendet wird (vgl. Davis 1989; Davis et al. 1989). Kim und Hyun (2016) konstatierten in diesem Zusammenhang, dass die erneute Nutzungsintention maßgeblich vom wahrgenommenen Nutzen einer virtuellen Anwendung abhängig ist. Übertragen auf den vorliegenden Forschungsgegenstand bedeutet dies, dass eine effiziente Beurteilung eines Leistungsangebotes mit Hilfe einer VR-Anwendung dazu führt, dass diese auch für zukünftige Leistungsbeurteilungen genutzt wird. Eine Effizienzsteigerung kann durch die Reduzierung von Unsicherheiten in der Planungsphase entstehen (Gursoy und McCleary 2004), wodurch der Entscheidungsfindungsprozess erleichtert wird (Hwang et al. 2013). Auf Basis der vorangegangenen Erkenntnisse ist davon auszugehen, dass der potenzielle Kunde mit Hilfe einer VR-Anwendung das Leistungsangebot besser beurteilen kann, wodurch die Nutzungseinstellung als auch die Nutzungsintention von VR-Anwendungen positiv beeinflusst werden. In Anbetracht dessen lassen sich folgende beiden Forschungshypothesen ableiten:

$\mathbf{H}_{1}$ Der wahrgenommene Nutzen einer VR-Anwendung hat einen positiven Einfluss auf die Nutzungseinstellung.

$\mathbf{H}_{2}$ Der wahrgenommene Nutzen einer VR-Anwendung hat einen positiven Einfluss auf die Nutzungsintention.

Eine einfache Bedienbarkeit des Informationssystems ist eine weitere Anforderung, die zu einer höheren Nutzerakzeptanz führt. Die Grundannahme dieser Wir- 
kungsbeziehung ist auf die Reduzierung von vorhandenen Bedienungsbarrieren zurückzuführen. Die einfache Bedienbarkeit spiegelt dabei den Grad der vom Nutzer empfundenen Anstrengungen, das Informationssystem zu nutzen, wider (Davis 1989; Davis et al. 1989). Je einfacher ein solches System vom Nutzer bedient werden kann, desto höher ist demnach die Wahrscheinlichkeit, dass der Nutzer das Informationssystem akzeptiert und somit zukünftig verwenden wird. Diese Auffassung basiert im Kern auf dem Prinzip des geringsten Aufwandes, wonach ein Individuum den einfachsten Weg zur Zielerreichung wählt (Zipf 2012). Bezogen auf VRAnwendungen bedeutet dies, dass die hardware- und softwareseitige Interaktion mit der Anwendung für den Konsument leicht zu erlernen, verständlich und intuitiv zu bedienen sein muss. Demzufolge muss sowohl das Design als auch die Usability der Anwendung ansprechend gestaltet und intuitiv zu bedienen sein, damit der Nutzer sich unmittelbar in der Anwendung orientieren kann. Diese Forderungen werden auch von aktuellen Forschungsergebnissen gestützt, etwa im Zusammenhang mit Augmented-Reality-Anwendungen, virtuellen Ankleidekabinen und Social-MediaAnwendungen (Chung et al. 2015; Kim und Forsythe 2008; Rauniar et al. 2014). Aufbauend auf den vorangegangenen Erkenntnissen lautet die zu überprüfende Hypothese wie folgt:

$\mathbf{H}_{3}$ Die wahrgenommene einfache Bedienbarkeit einer VR-Anwendung hat einen positiven Einfluss auf den wahrgenommenen Nutzen.

Darüber hinaus hat die wahrgenommene einfache Bedienbarkeit einen Einfluss auf die Nutzungseinstellung. Insbesondere bei Internetanwendungen ist die einfache Bedienbarkeit ein wesentliches Merkmal, das zu einer positiven Nutzungseinstellung führt (King und He 2006). Durch eine einfache Bedienbarkeit einer VR-Anwendung werden neben Bedienungs- ebenfalls Nutzungsbarrieren minimiert, wodurch die Nutzungseinstellung positiv beeinflusst wird. Eine positive Nutzungserfahrung, die aus einer einfachen Bedienbarkeit resultiert, prägt die Nutzungseinstellung gegenüber dem Informationssystem (Venkatesh 2000). Folglich kann die Nutzungseinstellung gegenüber einer VR-Anwendung positiv beeinflusst werden, wenn die Systemnutzung vom Nutzer als einfach erachtet wird. Ein derartiger Zusammenhang konnte bereits in zahlreichen Studien im Online-Umfeld nachgewiesen werden (Chung et al. 2015; Park 2009; Lee et al. 2006; Ayeh et al. 2013). Die Forschungshypothese lautet daher:

$\mathbf{H}_{4}$ Die wahrgenommene einfache Bedienbarkeit einer VR-Anwendung hat einen positiven Einfluss auf die Nutzungseinstellung.

Aufbauend auf der Theorie des überlegten Handelns ist die Nutzungseinstellung eine wichtige Determinante, die die zukünftige Nutzungsintention primär beeinflusst. Die Nutzungseinstellung ist in diesem Zusammenhang ein funktionaler Mediator, der die Überzeugung von einem Objekt widerspiegelt (Fishbein und Ajzen 1975). Übertragen auf VR-Anwendungen bedeutet dies, dass wenn der Nutzer der Überzeugung ist, dass die Nutzung der VR-Anwendung zu einem positiven Effekt führen könnte, sich auch die Bereitschaft erhöht, die Anwendung zu nutzen (Fishbein und Ajzen 1975; Bertrand und Bouchard 2008). Auch Rogers (2003) untermauert in der Diffusionstheorie diesen Effekt, wonach die Adaption einer Innovation durch 
die Nutzungseinstellung gegenüber der Innovation geprägt wird. Dementsprechend beeinflusst die individuelle Nutzungseinstellung die Nutzungsintention, sodass auf Basis dieser Wirkungsbeziehung die Entscheidung für oder gegen die Adaption der Innovation getroffen wird (Rogers 2003). In Anbetracht dessen lautet die zu verifizierende Forschungshypothese wie folgt:

H5 $_{5}$ Die Nutzungseinstellung gegenüber einer VR-Anwendung hat einen positiven Einfluss auf die Nutzungsintention.

\subsection{Telepräsenz}

VR-Anwendungen können dem Nutzer das Gefühl vermitteln, sich tatsächlich an einem anderen Ort zu befinden. Dieses Gefühl wird in der Literatur als Telepräsenz bezeichnet (Klein 2003; Steuer 1992). Telepräsenz repräsentiert den Grad des vom Nutzer wahrgenommenen Gefühls, sich eher in der virtuellen als in der physikalischen Realität zu befinden (Steuer 1992; Sanchez-Vives und Slater 2005).

Bei klassischen web-basierten Produktpräsentationen intensiviert sich das Produkterlebnis, wenn der Anwender direkt mit der Produktpräsentation interagieren kann (Klein 2003). Die direkte Interaktion mit der Produktpräsentation verstärkt beim Nutzer das Gefühl, sich an einem anderen Ort zu befinden, wodurch eine reale Produktprüfung teilweise substituiert wird (Klein 2003). Diesbezüglich zeigen Untersuchungen, dass die Intensität der Immersion einen positiven Einfluss auf die Informationsaufnahme und -verarbeitung hat und die Produktbeurteilung erleichtert (Choi et al. 2016). Mithilfe von VR-Anwendungen ist es möglich, ein realistisches, interaktives Abbild einer Leistung zu erzeugen. Gemäß der Cognitive-FitTheory wird die Produktbeurteilung (Problem) durch die neuartigen Visualisierungsmöglichkeiten (Problemrepräsentation) von VR-Anwendungen erheblich erleichtert, sodass nur ein geringer kognitiver Aufwand für die Problemlösung notwendig ist (Vessey 1991). Bei Reiseangeboten beispielsweise entfällt der kognitive Aufwand sich eine Reiseunterkunft auf Grundlage von Katalogbildern imaginär vorstellen zu müssen, da sich der potenzielle Kunde mittels einer VR-Anwendung direkt einen realistischen Eindruck verschaffen kann. Das von VR-Anwendungen erzeugte immersive Gefühl ermöglicht es dem potenziellen Kunden, relevante Produktinformationen schneller und einfacher zu erfassen, was zu einer effizienteren Gesamtbeurteilung führt und dadurch den wahrgenommenen Nutzen der Anwendung erhöht. Des Weiteren werden durch einen hohen Grad der Telepräsenz eine realitätsnahe, intuitive Navigation und Interaktion ermöglicht, vorausgesetzt die VR-Anwendung wurde entsprechend gut gestaltet. Die zu verifizierenden Forschungshypothesen lauten daher wie folgt:

Ho Die Telepräsenz hat einen positiven Einfluss auf den wahrgenommenen Nutzen.

$\mathbf{H}_{7}$ Die Telepräsenz hat einen positiven Einfluss auf die wahrgenommene einfache Bedienbarkeit.

Abb. 1 fasst das konzeptionelle Forschungsmodell nochmal zusammen. 


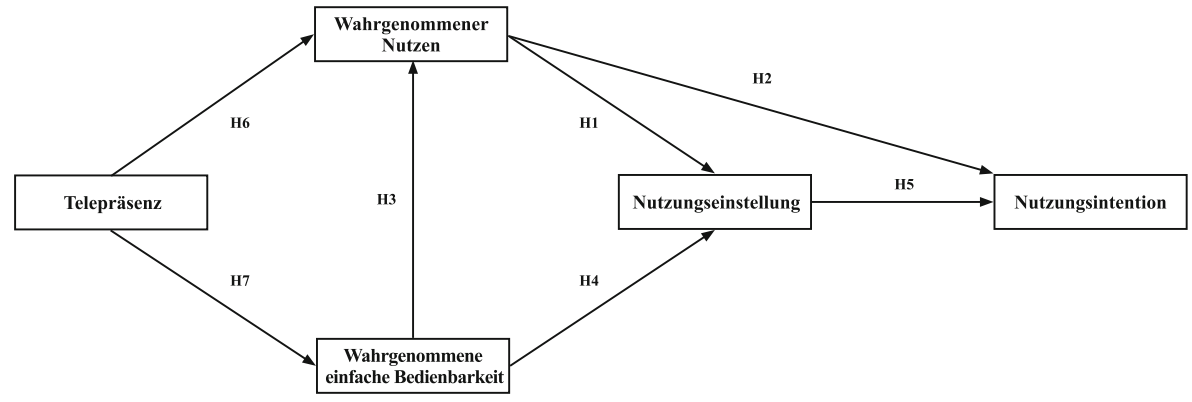

Abb. 1 Konzeptionelles Forschungsmodell

\section{Methodik}

\subsection{Operationalisierung}

Die Konstrukte wurden auf Basis von etablierten, empirisch validierten Indikatoren operationalisiert. Sowohl das Konstrukt des wahrgenommenen Nutzens (5 Fragen), als auch die wahrgenommene einfache Bedienbarkeit (4 Fragen) wurden von Davis (1989) adaptiert und auf den vorliegenden Forschungsgegenstand übertragen. Gleiches gilt für die Operationalisierung der Nutzungseinstellung (3 Fragen), welche in Anlehnung an Venkatesh et al. (2003) erfolgte. Die Bewertung der Nutzungsintention wird anhand von drei Indikatoren gemessen, die aus den Studien von Venkatesh (2000) und Lee und Lehto (2013) abgeleitet sind. Basierend auf der Studie von Klein (2003) wird das Gefühl der Telepräsenz durch drei Indikatoren operationalisiert. Die Bewertung der einzelnen Indikatoren erfolgte mittels einer 7-Punkt-LikertSkala, deren Skalenpole von ,trifft überhaupt nicht zu“ (1) bis ,trifft voll und ganz zu“ (7) reichte.

\subsection{Entwicklung der VR-Anwendung und Design der Untersuchung}

Zur Überprüfung der Hypothesen wurde eine experimentelle Untersuchung durchgeführt. Als Untersuchungsgegenstand wurde eine touristische Unterkunft (Hotel) gewählt, da mit einem solchem Erfahrungsgut sehr viele Individuen schon einmal Berührungspunkte hatten. Da VR-Anwendungen ein immer noch recht neues Anwendungsfeld sind, konnte nicht davon ausgegangen werden, dass alle Probanden das Potenzial dieser Technologie bewerten können. Daher wurde eigens für die Studie eine Anwendung entwickelt, in welcher die Probanden ein Hotel virtuell erkunden konnten.

Das Experiment untergliederte sich in vier Phasen. In einem Einführungsvideo wurde den Probanden in Phase eins die VR-Anwendung und der Ablauf der Studie vorgestellt. Im Anschluss wurden in der zweiten Phase die soziodemografischen Daten der Studienteilnehmer und die bisherigen Erfahrungen mit VR-Anwendungen erfasst. Zu Beginn der dritten Phase erhielten die Probanden eine VR-Brille, um sich ein Demonstrationsvideo anzusehen. Nach der Demonstration startete eine Audiowiedergabe, in welcher das Szenario beschrieben wurde. In diesem Szenario sollten 
sich die Probanden vorstellen, dass sie eine zweiwöchige All-inclusive-Reise planen und sich mithilfe einer VR-Anwendung über ein Hotel informieren. Anschließend konnten die Probanden ein Hotel mittels der VR-Anwendung frei erkunden. Die maximale Nutzungsdauer der VR-Anwendung wurde auf zehn Minuten begrenzt, jedoch konnten die Probanden jederzeit, durch das Absetzen der VR-Brille, die Tour beenden. In der vierten Phase wurde den Studienteilnehmern ein zweiter Fragebogen ausgehändigt, in welchem sie die VR-Anwendung beurteilen sollten.

\subsection{Datenerhebung und -analyse}

Zur Validierung des Fragebogens und der VR-Anwendung wurde ein Pretest durchgeführt. Daraufhin wurden einige Indikatoren des Fragebogens weiter konkretisiert. Zur Gewinnung der Probanden wurden unterschiedliche Quellen genutzt. Neben Angestellten von Unternehmen und öffentlichen Institutionen wurden Studierende und Mitarbeiter einer deutschen Hochschule eingeladen, an der Studie teilzunehmen. Als Anreiz wurde unter allen Studienteilnehmern ein Tablet verlost. Die Untersuchung wurde im ersten Quartal 2017 durchgeführt. Während des Untersuchungszeitraums nahmen insgesamt 569 Probanden teil und es konnten 540 gültige Datensätze erhoben werden. Zur Modellschätzung wurde in der vorliegenden Studie das PLS-SEM Verfahren genutzt, welches sich speziell im Kontext neuartiger Technologien für die Modellierung von latenten Variablen eignet (Henseler et al. 2016).

\section{Ergebnisse}

\subsection{Stichprobenmerkmale}

Von den gültigen Studienteilnehmern waren 54,3\% männlich und 45,7\% weiblich. Die am stärksten vertretene Altersgruppe waren die 20- bis 29-Jährigen (58,3\%). Der Begriff ,Virtual-Reality“ war nahezu allen Probanden (93,7\%) vor der Untersuchung bekannt und 50,2\% haben bereits eine VR-Anwendung genutzt. Circa $60 \%$ der Probanden waren Studierende und $30 \%$ Angestellte.

\subsection{Beurteilung des Messmodells}

Die Beurteilung des Messmodells erfolgt anhand der internen Konsistenz, der Konvergenz- sowie der Diskriminanzvalidität (Hair et al. 2017). Die Reliabilitätsprüfung der internen Konsistenz wird mittels dreier Kriterien durchgeführt (Henseler et al. 2016). Neben dem Cronbach's Alpha Koeffizienten $(\alpha)$, der Faktorreliabilität $\left(\rho_{c}\right)$ wird ebenfalls der Dijkstra-Henseler's Koeffizient $\left(\rho_{\mathrm{A}}\right)$ für die Verifizierung der internen Konsistenz herangezogen. Wie Tab. $1 \mathrm{zu}$ entnehmen ist, befinden sich alle Werte über den geforderten Mindestanforderungen der jeweiligen Kriterien, sodass eine interne Konsistenz gegeben ist.

Die Konvergenzvalidität des Messmodells wird in der vorliegenden Untersuchung mittels der Faktorenladungen, der Indikatorreliabilität sowie der durchschnittlich erfassten Varianz (DEV) überprüft. Ein Beleg für die Konvergenzvalidität des Messmo- 
Tab. 1 Validität und Reliabilität der Konstrukte

\begin{tabular}{|c|c|c|c|c|c|}
\hline Konstrukte und Indikatoren & $\alpha$ & $\rho \mathrm{A}$ & $\rho c$ & DEV & Ladung \\
\hline Kriterien & $>0,7$ & $>0,7$ & $>0,7$ & $>0,5$ & $>0,7$ \\
\hline Telepräsenz (TELE) & $\mathbf{0 , 8 2 0}$ & 0,845 & $\mathbf{0 , 8 9 3}$ & 0,737 & \\
\hline \multicolumn{5}{|c|}{$\begin{array}{l}\text { Während ich die Virtual-Reality-Anwendung genutzt habe, hat es sich so angefühlt, als ob } \\
\text { ich in einer anderen Welt bin }\end{array}$} & 0,763 \\
\hline \multicolumn{5}{|c|}{ Durch die virtuelle Simulation hatte ich das Gefühl, die Situation wirklich zu erleben } & 0,896 \\
\hline \multicolumn{5}{|c|}{$\begin{array}{l}\text { Als ich durch die virtuelle Welt gegangen bin, hatte ich das Gefühl an einem anderen Ort zu } \\
\text { sein }\end{array}$} & 0,909 \\
\hline Wahrgenommener Nutzen (WN) & $\mathbf{0 , 8 8 7}$ & $\mathbf{0 , 8 9 5}$ & 0,917 & $\mathbf{0 , 6 8 9}$ & \\
\hline \multicolumn{5}{|c|}{$\begin{array}{l}\text { Durch die Virtual-Reality-Anwendung kann ich mir schneller einen Eindruck vom Hotel } \\
\text { verschaffen }\end{array}$} & 0,757 \\
\hline \multicolumn{5}{|c|}{ Durch die Virtual-Reality-Anwendung kann ich einfacher das Hotel beurteilen } & 0,817 \\
\hline \multicolumn{5}{|c|}{ Durch die Nutzung der Virtual-Reality-Anwendung kann ich das Hotel besser beurteilen } & 0,844 \\
\hline \multicolumn{5}{|c|}{ Ich finde die Virtual-Reality-Anwendung sinnvoll, um mir ein Hotel anzusehen } & 0,848 \\
\hline \multicolumn{5}{|c|}{$\begin{array}{l}\text { Insgesamt finde ich, dass die Virtual-Reality-Anwendung nützlich ist, um mir einen Ein- } \\
\text { druck vom Hotel zu verschaffen }\end{array}$} & 0,878 \\
\hline $\begin{array}{l}\text { Wahrgenommene einfache Bedienbarkeit } \\
\text { (WEB) }\end{array}$ & $\mathbf{0 , 8 3 1}$ & $\mathbf{0 , 8 4 7}$ & $\mathbf{0 , 8 8 7}$ & 0,663 & \\
\hline \multicolumn{5}{|c|}{ Die Handhabung der Virtual-Reality-Anwendung war einfach für mich } & 0,824 \\
\hline \multicolumn{5}{|c|}{ Ich fand es schwer, die Virtual-Reality-Anwendung zu bedienen ${ }^{\mathrm{a}}$} & 0,764 \\
\hline \multicolumn{5}{|c|}{ Die Bedienung der Virtual-Reality-Anwendung war einfach } & 0,791 \\
\hline \multicolumn{5}{|c|}{ Insgesamt finde ich die Virtual-Reality-Anwendung einfach zu benutzen } & 0,874 \\
\hline Nutzungseinstellung (NE) & $\mathbf{0 , 9 2 8}$ & $\mathbf{0 , 9 2 9}$ & 0,954 & $\mathbf{0 , 8 7 5}$ & \\
\hline \multicolumn{6}{|l|}{ Ich bin der Meinung, dass die VR-Anwendung ... } \\
\hline \multicolumn{5}{|c|}{ Eine sehr interessante Technologie ist, um sich ein Hotel anzusehen } & 0,925 \\
\hline \multicolumn{5}{|l|}{ Eine gute Idee ist, um sich ein Hotel anzusehen } & 0,949 \\
\hline \multicolumn{5}{|c|}{ Eine tolle Möglichkeit ist, um sich einen Eindruck von einem Hotel zu verschaffen } & 0,932 \\
\hline Nutzungsintention (NI) & $\mathbf{0 , 8 9 0}$ & 0,904 & 0,924 & 0,754 & \\
\hline \multicolumn{6}{|c|}{ Vorausgesetzt ich würde eine VR-Brille besitzen, dann würde ich sie ... } \\
\hline \multicolumn{5}{|l|}{ Benutzen, um mir ein Hotel virtuell anzusehen } & 0,911 \\
\hline \multicolumn{5}{|c|}{ Dazu verwenden, um mir Informationen zu einem Hotel einzuholen } & 0,754 \\
\hline \multicolumn{5}{|c|}{ Nutzen, um mir einen Eindruck vom Hotel zu verschaffen } & 0,929 \\
\hline \multicolumn{5}{|c|}{ Anderen weiterempfehlen, die sich einen Eindruck vom Hotel machen möchten } & 0,869 \\
\hline
\end{tabular}

${ }^{\text {a}}$ Reverse-Coded

dells sind hohe Faktorladungen jener Indikatoren, die einem Konstrukt zugeordnet sind. Diesbezüglich sollten die äußeren Faktorladungen der zugeordneten Indikatoren einen Wert von 0,707 überschreiten (Hair et al. 2017). Wie die Tab. 1 aufzeigt, wird dieses Kriterium von allen Indikatoren erfüllt. Darüber hinaus kann durch die Quadrierung der einzelnen Faktorenladungen die jeweilige Indikatorreliabilität $(>0,5)$ ermittelt werden (Hair et al. 2017). Folglich wird die Indikatorreliabilität bestätigt, da alle Faktorladungen sich über dem Grenzwert von 0,707 befinden. Gleiches gilt für die DEV, die bei allen Konstrukten über der Mindestanforderung von 0,5 (Henseler et al. 2016) liegt (vgl. Tab. 1). 
Tab. 2 Quadrierte Korrelation zwischen den Konstrukten (DEV siehe Diagonale) und HTMT.85 Kriterium (kursiv)

\begin{tabular}{llllll}
\hline & TELE & WN & WEB & NE & NI \\
\hline TELE & $\mathbf{0 , 7 3 7}$ & 0,533 & 0,279 & 0,445 & 0,493 \\
WN & 0,209 & $\mathbf{0 , 6 8 9}$ & 0,229 & 0,776 & 0,800 \\
WEB & 0,057 & 0,040 & $\mathbf{0 , 6 6 3}$ & 0,235 & 0,182 \\
NE & 0,147 & 0,507 & 0,044 & $\mathbf{0 , 8 7 5}$ & 0,719 \\
NI & 0,177 & 0,516 & 0,026 & 0,438 & $\mathbf{0 , 7 5 4}$ \\
\hline
\end{tabular}

Die Diskriminanzvalidität beschreibt, inwieweit sich ein Konstrukt von den anderen Konstrukten des Messmodells unterscheidet. Für die erste Beurteilung der Diskriminanzvalidität werden die Kreuzladungen der einzelnen Indikatoren betrachtet. In diesem Zusammenhang sollte ein Indikator die höchste Faktorladung mit dem ihm zugeordneten Konstrukt aufweisen. Diese Anforderung wurde von allen Indikatoren erfüllt. Ein weiteres Verfahren, um die Diskriminanzvalidität des Messmodells zu bestimmen, stellt das Fornell-Larcker Kriterium (Fornell und Larcker 1981) dar. Bei diesem Kriterium muss die DEV eines Konstrukts höher als die quadrierten Interkorrelationen mit den anderen Konstrukts sein. Die Ergebnisse in Tab. 2 belegen, dass die Diskriminanzvalidität durch das Fornell-Larcker Kriterium bestätigt werden kann. Zusätzlich wurde die Diskriminanzvalidität mithilfe des Heterotrait-monotrait (HTMT) ratio of correlations verifiziert (Henseler et al. 2015). In der vorliegenden Untersuchung wird zur Bestimmung der Diskriminanzvalidität der konservative Grenzwert HTMT.85 gewählt (Henseler et al. 2015). Wie der Tab. 2 zu entnehmen ist, kann die Diskriminanzvalidität des Messmodells ebenfalls durch das HTMT-Verfahren bestätigt werden, da alle Ergebnisse den Grenzwert von 0,85 unterschreiten.

\subsection{Beurteilung des Strukturmodells}

Die Beurteilung des Strukturmodells erfolgt anhand des Bestimmtheitsmaßes $\left(\mathrm{R}^{2}\right)$, der Prognoserelevanz $\left(\mathrm{Q}^{2}\right)$, dem Standardized Root Mean Square Residual (SRMR), der Stärke der Pfadkoeffizienten sowie der Signifikanz der hypothetischen Wirkungsbeziehungen. Das Bestimmtheitsmaß $\left(\mathrm{R}^{2}\right)$ wird häufig für die Beurteilung des Strukturmodells verwendet und spiegelt den Anteil der erklärten Varianz wider (Hair et al. 2017). Die Interpretation der $\mathrm{R}^{2}$-Werte für endogene Variablen erfolgt auf Basis der von Chin (1998) vorgeschlagenen Skala. Demzufolge wird der Anteil der erklärten Varianz ab 0,19 als schwach, ab 0,33 als moderat und ab 0,67 als substantiell angesehen (Chin 1998). Mit zwei Ausnahmen befinden sich alle endogenen Variablen (Abb. 2) über dem Schwellenwert von 0,33, der für eine moderate Modellbeurteilung gefordert wird.

Um die Prognoserelevanz $\left(\mathrm{Q}^{2}\right)$ des Strukturmodells $\mathrm{zu}$ bestimmen, wurde der Stone-Geisser Test (Stone 1974; Geisser 1974) auf Basis des kreuzvalidierten Redundanz-Ansatzes durchgeführt. Mittels der Blindfolding-Prozedur werden die empirisch erhobenen Daten der endogenen Variablen systematisch im Ursprungsdatensatz als fehlend angenommen und durch Schätzparameter des aufgestellten Modells er- 


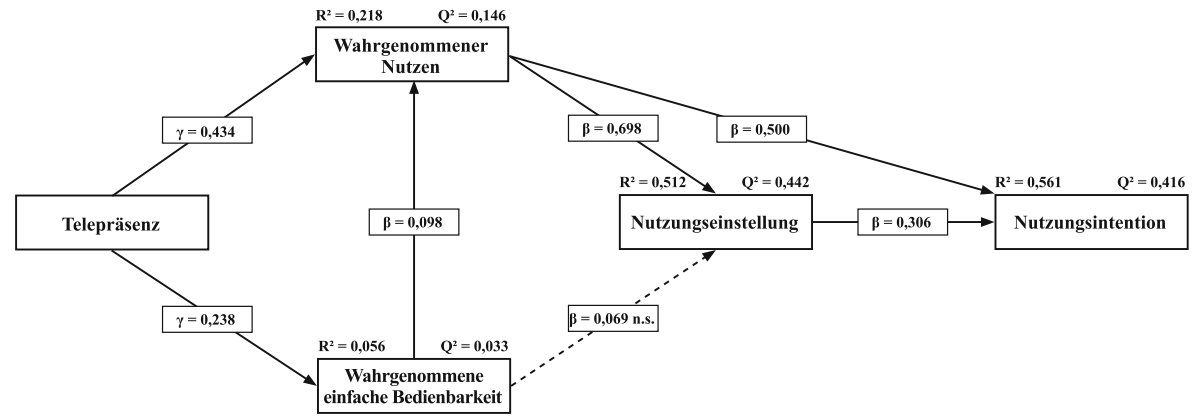

Abb. 2 PLS-Ergebnisse des Strukturgleichungsmodells

setzt (Hair et al. 2017). Somit kann durch den Stone-Geisser Test ermittelt werden, ob das aufgestellte Modell für die Rekonstruktion empirischer Daten geeignet ist $\left(Q^{2}>0\right)$ (Chin 1998). Wie aus Abb. 2 hervorgeht, wird dieses Kriterium von allen endogenen Variablen erfüllt, sodass die Prognosefähigkeit des Modells konstatiert werden kann. Darüber hinaus weist der Standardized Root Mean Square Residual (SRMR) einen Wert von 0,058 auf, welcher sich unter dem Grenzwert von 0,08 befindet, sodass die Anpassungsgüte des Modells bestätigt wird (Hair et al. 2017; Henseler et al. 2016).

Abschließend werden die Pfadkoeffizienten des Strukturmodells analysiert, die zur Überprüfung der Forschungshypothesen notwendig sind. Während die Stärke der Pfadkoeffizienten direkt durch den PLS-Algorithmus berechnet wird, muss die Signifikanz der Pfadkoeffizienten separat anhand des Bootstrapping-Verfahrens verifiziert werden. Wie die Berechnung der Pfadkoeffizienten aufzeigt, stimmen nahezu alle Strukturpfade mit den angenommenen Wirkungsbeziehungen überein (Tab. 3). Einzig die Stärke der Wirkungsbeziehungen zwischen der wahrgenommenen einfachen Bedienbarkeit und dem wahrgenommenen Nutzen sowie der Nutzungseinstellung sind nur schwach ausgeprägt. Um die Signifikanz der Pfadkoeffizienten zu ermitteln, wurde ein vollständiges Bootstrapping (5000 Untergruppen) durchgeführt. Die Ergebnisse der Bootstrapping-Prozedur sind in Tab. 3 aufgeführt. Insgesamt konnte die statistische Signifikanz der Pfadkoeffizienten für sechs der sieben angenommenen Wirkungsbeziehungen nachgewiesen werden. Demnach wirkt sich

Tab. 3 Ergebnisse der Hypothesentests

\begin{tabular}{lllllll}
\hline & Hypothese & Pfadkoeffizient & $\begin{array}{l}\text { Konfidenzintervalle } \\
\text { (Bias korrigiert) }\end{array}$ & t-Wert & $p$-Wert & Unterstützt \\
\hline H1 & WN $\rightarrow$ NE & $0,698^{* * *}$ & {$[0,625 ; 0,759]$} & 20,461 & 0,000 & Ja \\
H2 & WN $\rightarrow$ NI & $0,500^{* * *}$ & {$[0,400,0,597]$} & 9,968 & 0,000 & Ja \\
H3 & WEB $\rightarrow$ WN & $0,098^{*}$ & {$[-0,001 ; 0,197]$} & 1,970 & 0,049 & Ja \\
H4 & WEB $\rightarrow$ NE & 0,069 & {$[-0,003 ; 0,142]$} & 1,883 & 0,060 & Nein \\
H5 & NE $\rightarrow$ NI & $0,306 * * *$ & {$[0,203 ; 0,413]$} & 5,671 & 0,000 & Ja \\
H6 & TELE $\rightarrow$ WN & $0,434 * * *$ & {$[0,345 ; 0,514]$} & 10,139 & 0,000 & Ja \\
H7 & TELE $\rightarrow$ WEB & $0,238^{* * *}$ & {$[0,148 ; 0,324]$} & 5,252 & 0,000 & Ja \\
\hline
\end{tabular}

$* * * p<0,001, * p<0,05$ 
der wahrgenommene Nutzen einer VR-Anwendung positiv auf die Nutzungseinstellung $(\beta=0,698, t=20,461, p<0,001)$ sowie zukünftige Nutzungsintention $(\beta=0,500$, $\mathrm{t}=9,968, p<0,001)$ aus. Des Weiteren bestätigte sich ein signifikanter Wirkungszusammenhang zwischen der Nutzungseinstellung und -intention $(\beta=0,306, t=5,671$, $p<0,001)$. Der wahrgenommene Nutzen wird durch das Konstrukt Telepräsenz $(\gamma=0,434, t=10,139, p<0,001)$ positiv beeinflusst. Dies gilt auch für die wahrgenommene einfache Bedienbarkeit $(\gamma=0,238, \mathrm{t}=5,252, p<0,001)$. Die wahrgenommene einfache Bedienbarkeit weist lediglich eine schwache, aber dennoch signifikante Wirkungsbeziehung mit dem wahrgenommenen Nutzen $(\beta=0,098, t=1,970$, $p<0,05)$ auf, wohingegen die Wirkungsbeziehung zwischen der wahrgenommenen einfachen Bedienbarkeit und der Nutzungseinstellung $(\beta=0,069, \mathrm{t}=1,883, p>0,06)$ nicht bestätigt werden konnte.

\section{Schlussbetrachtung}

Basierend auf dem TAM wurde in der vorliegenden Studie sowohl der wahrgenommene Nutzen als auch die wahrgenommene einfache Bedienbarkeit hinsichtlich ihres Effekts auf die Nutzungseinstellung und -intention von VR-Anwendungen untersucht. Zusätzlich wurde die Bedeutung der Telepräsenz, als zentrales Merkmal von VR-Anwendungen, und deren Einfluss auf den wahrgenommenen Nutzen sowie die wahrgenommene einfache Bedienbarkeit einer VR-Anwendung analysiert.

Wie die Ergebnisse verdeutlichen, ist speziell der wahrgenommene Nutzen ein zentraler Prädiktor, der sowohl die Nutzungseinstellung als auch die Nutzungsintention positiv beeinflusst. Gleichzeitig weist unsere Studie die signifikante Wirkungsbeziehung zwischen Nutzungseinstellung und -intention nach, die auch im Kontext mit anderen Technologien identifiziert werden konnte (Chung et al. 2015; Cheng und Cho 2011; Ayeh et al. 2013). Neben den klassischen Einflussfaktoren des TAM wurde in der vorliegenden Studie ebenfalls die Wirkung der Telepräsenz auf den wahrgenommenen Nutzen und die wahrgenommene einfache Bedienbarkeit untersucht. In beiden Fällen zeigen die Ergebnisse einen positiven signifikanten Einfluss. Diese Ergebnisse stehen im Einklang mit vorangegangenen Forschungsarbeiten, die im Kontext mit virtuellen Produktpräsentationen durchgeführt wurden (Klein 2003; Choi et al. 2016). Durch die realitätsgetreuen Visualisierungsmöglichkeiten sind VR-Anwendungen dazu in der Lage, das virtuelle Gegenwartsempfinden der Nutzer positiv zu beeinflussen. Dies ermöglicht eine einfache und intuitive Navigation sowie Interaktion. Dadurch können potenzielle Kunden relevante Produktinformationen schneller und einfacher erfassen. Ein wesentlicher Vorteil dieser Technologie besteht somit in einer effizienteren Informationsbeschaffung, die eine fundierte Entscheidungsfindung ermöglicht. Durch die Vielzahl der zur Verfügung stehenden Kommunikationskanäle müssen Unternehmen eine nutzerzentrierte Perspektive einnehmen, um die Informationen über den aus Nutzersicht geeigneten Kanal zu kommunizieren. Wie die Ergebnisse unserer Studie verdeutlichen, sind VR-Anwendungen ein adäquates Marketinginstrument, das zur Informationskommunikation eingesetzt werden kann. Im Rahmen der vorliegenden Untersuchung wurden keine spezifischen gestalterischen sowie technischen Rahmenbedingungen 
der VR-Anwendung wie beispielsweise der Aufbau des Interaktionsdesigns oder die Qualität des Bildmaterials thematisiert. Diese Aspekte wurden in vorangegangenen Forschungsarbeiten untersucht (Chen et al. 2020; Hepperle et al. 2019; Flavián et al. 2019).

Insbesondere für erklärungsbedürftige Produkte bietet die VR-Technologie neuartige Visualisierungsmöglichkeiten. Die potenziellen Kunden können im Vorfeld des Kaufes ein Produkt beziehungsweise eine Dienstleistung besser beurteilen, sodass das Risiko einer informationsbedingten Fehlentscheidung reduziert wird. Diese Erkenntnis ist insbesondere für Anbieter hochpreisiger Erfahrungsgüter, beispielsweise in der Tourismusbranche, von großer Bedeutung. Vor allem das Gefühl, sich unmittelbar vor Ort zu befinden, verringert die inhärente Informationsasymmetrie solcher Erfahrungsgüter und unterstützt die potenziellen Kunden bei der Leistungsbeurteilung. Durch den Einsatz von VR-Anwendungen können Marketingverantwortliche somit den Informationsbeschaffungsprozess der Kunden verkürzen. VR-Anwendungen versetzen die Anbieter folglich in die Lage, den Kaufentscheidungsprozess der potenziellen Kunden positiv zu beeinflussen.

Funding Open Access funding enabled and organized by Projekt DEAL.

Open Access Dieser Artikel wird unter der Creative Commons Namensnennung 4.0 International Lizenz veröffentlicht, welche die Nutzung, Vervielfältigung, Bearbeitung, Verbreitung und Wiedergabe in jeglichem Medium und Format erlaubt, sofern Sie den/die ursprünglichen Autor(en) und die Quelle ordnungsgemäß nennen, einen Link zur Creative Commons Lizenz beifügen und angeben, ob Änderungen vorgenommen wurden.

Die in diesem Artikel enthaltenen Bilder und sonstiges Drittmaterial unterliegen ebenfalls der genannten Creative Commons Lizenz, sofern sich aus der Abbildungslegende nichts anderes ergibt. Sofern das betreffende Material nicht unter der genannten Creative Commons Lizenz steht und die betreffende Handlung nicht nach gesetzlichen Vorschriften erlaubt ist, ist für die oben aufgeführten Weiterverwendungen des Materials die Einwilligung des jeweiligen Rechteinhabers einzuholen.

Weitere Details zur Lizenz entnehmen Sie bitte der Lizenzinformation auf http://creativecommons.org/ licenses/by/4.0/deed.de.

\section{Literatur}

Ayeh JK, Au N, Law R (2013) Predicting the intention to use consumer-generated media for travel planning. Tour Manag 35:132-143. https://doi.org/10.1016/j.tourman.2012.06.010

Bertrand M, Bouchard S (2008) Applying the technology acceptance model to vr with people who are favorable to its use. J Cyberther Rehabil 1(2):200-210

Buhalis D, Law R (2008) Progress in information technology and tourism management: 20 years on and 10 years after the internet-the state of eTourism research. Tour Manag 29(4):609-623. https://doi. org/10.1016/j.tourman.2008.01.005

Chen M, Jin Y, Goodall T, Yu X, Bovik AC (2020) Study of 3D virtual reality picture quality. IEEE J Sel Top Signal Process 14:89-102. https://doi.org/10.1109/JSTSP.2019.2956408

Cheng S, Cho V (2011) An integrated model of employees' behavioral intention toward innovative information and communication technologies in travel agencies. J Hosp Tour Res 35(4):488-510. https:// doi.org/10.1177/1096348010384598

Chin WW (1998) The partial least squares approach for structural equation modeling. In: Marcoulides GA (Hrsg) Modern methods for business research. Lawrence Erlbaum, Mahwah, S 295-336

Choi J, Ok C, Choi S (2016) Outcomes of destination marketing organization website navigation: the role of telepresence. J Travel Tour Mark 33(1):46-62. https://doi.org/10.1080/10548408.2015.1024913 
Chung N, Han H, Joun Y (2015) Tourists' intention to visit a destination: the role of augmented reality (AR) application for a heritage site. Comput Hum Behav 50:588-599. https://doi.org/10.1016/j.chb. 2015.02.068

Davis FD (1989) Perceived usefulness, perceived ease of use, and user acceptance of information technology. Mis Q 13(3):319-340. https://doi.org/10.2307/249008

Davis FD, Bagozzi RP, Warshaw PR (1989) User acceptance of computer technology: a comparison of two theoretical models. Manag Sci 35:982-1003. https://doi.org/10.1287/mnsc.35.8.982

diginetmedia (2021) Reiseveranstalter mit Virtual Reality-Anwendungen. https://diginetmedia.de/ virtualreality/. Zugegriffen: 7. Sept. 2021

Dowling GR, Staelin R (1994) A model of perceived risk and intended risk-handling activity. J Consum Res 21(1):119-134. https://doi.org/10.1086/209386

Fishbein M, Ajzen I (1975) Belief, attitude, intention and behavior: an introduction to theory and research. Addison-Wesley series in social psychology. Addison-Wesley, Reading

Flanagin AJ, Metzger MJ, Pure R, Markov A, Hartsell E (2014) Mitigating risk in ecommerce transactions: perceptions of information credibility and the role of user-generated ratings in product quality and purchase intention. Electron Commer Res 14(1):1-23. https://doi.org/10.1007/s10660-014-9139-2

Flavián C, Ibáñez-Sánchez S, Orús C (2019) The impact of virtual, augmented and mixed reality technologies on the customer experience. J Bus Res 100:547-560. https://doi.org/10.1016/j.jbusres.2018.10. 050

Fornell C, Larcker DF (1981) Evaluating structural equation models with unobservable variables and measurement error. J Mark Res 18(1):39-50. https://doi.org/10.2307/3151312

Geisser S (1974) A predictive approach to the random effect model. Biometrika 61(1):101. https://doi.org/ $10.2307 / 2334290$

Gursoy D, McCleary KW (2004) An integrative model of tourists' information search behavior. Ann Tour Res 31(2):353-373. https://doi.org/10.1016/j.annals.2003.12.004

Hair JF, Hult GTM, Ringle CM, Sarstedt M (2017) A primer on partial least squares structural equations modeling (PLS-SEM), 2. Aufl. SAGE, Los Angeles

Häubl G, Trifts V (2000) Consumer decision making in Online shopping environments: the effects of interactive decision aids. Mark Sci 19(1):4-21. https://doi.org/10.1287/mksc.19.1.4.15178

Henseler J, Ringle CM, Sarstedt M (2015) A new criterion for assessing discriminant validity in variancebased structural equation modeling. J Acad Mark Sci 43(1):115-135. https://doi.org/10.1007/s11747014-0403-8

Henseler J, Hubona G, Ray PA (2016) Using PLS path modeling in new technology research: updated guidelines. Ind Manag Data Syst 116(1):2-20. https://doi.org/10.1108/IMDS-09-2015-0382

Hepperle D, Weiß Y, Siess A, Wölfel M (2019) 2D, 3D or speech? A case study on which user interface is preferable for what kind of object interaction in immersive virtual reality. Comput Graph 82:321-331. https://doi.org/10.1016/j.cag.2019.06.003

Hollebeek LD, Clark MK, Andreassen TW, Sigurdsson V, Smith D (2020) Virtual reality through the customer journey: Framework and propositions. J Retail Consum Serv. https://doi.org/10.1016/j. jretconser.2020.102056

Hwang YH, Jani D, Jeong HK (2013) Analyzing international tourists' functional information needs: a comparative analysis of inquiries in an on-line travel forum. J Bus R 66(6):700-705. https://doi.org/ 10.1016/j.jbusres.2011.09.006

Isaac J (2016) Step into a new world: virtual reality. https://www.completegate.com/2016070154/blog/ virtual-reality-explained. Zugegriffen: 20. März 2021

Kaplanidou K, Vogt C (2006) A structural analysis of destination travel intentions as a function of web site features. J Travel Res 45(2):204-216. https://doi.org/10.1177/0047287506291599

Kim HC, Hyun MY (2016) Predicting the use of smartphone-based augmented reality (AR): does telepresence really help? Comput Hum Behav 59:28-38. https://doi.org/10.1016/j.chb.2016.01.001

Kim J, Forsythe S (2008) Adoption of virtual try-on technology for online apparel shopping. J Interact Mark 22(2):45-59. https://doi.org/10.1002/dir.20113

King WR, He J (2006) A meta-analysis of the technology acceptance model. Inf Manag 43(6):740-755. https://doi.org/10.1016/j.im.2006.05.003

Klein LR (2003) Creating virtual product experiences: the role of telepresence. J Interact Mark 17(1):41-55. https://doi.org/10.1002/dir.10046

Lee DY, Lehto MR (2013) User acceptance of youtube for procedural learning: an extension of the technology acceptance model. Comput Educ 61:193-208. https://doi.org/10.1016/j.compedu.2012.10.001 
Lee H, Fiore AM, Kim J (2006) The role of the technology acceptance model in explaining effects of image interactivity technology on consumer responses. Int J Retail Distrib Manag 34(8):621-644. https://doi.org/10.1108/09590550610675949

Legris P, Ingham J, Collerette P (2003) Why do people use information technology?: A critical review of the technology acceptance model. Inf Manag 40(3):191-204. https://doi.org/10.1016/S03787206(01)00143-4

Li H, Daugherty T, Biocca F (2001) Characteristics of virtual experience in electronic commerce: a protocol analysis. J Interact Mark 15(3):13-30. https://doi.org/10.1002/dir.1013

Manis K, Choi D (2019) The virtual reality hardware acceptance model (VR-HAM): Extending and individuating the technology acceptance model (TAM) for virtual reality hardware. J Bus Res 100:503-513. https://doi.org/10.1016/j.jbusres.2018.10.021

Omdia (2020) Consumer VR headset and content revenue forecast 2020-2025. https://omdia.tech.informa. com/pr/2020-dec/six-and-a-half-million-consumer-vr-headsets-will-be-sold-in-2020. Zugegriffen: 7. Sept. 2021

Park SY (2009) An analysis of the technology acceptance model in understanding university students' behavioral intention to use e-learning. J Educ Techno Soc 12(3):150-162

PWC (2018) Growing VR/AR companies in the UK. https://www.pwc.co.uk/intelligent-digital/vr/ growing-vr-ar-companies-in-the-uk.pdf. Zugegriffen: 25. März 2021

Rauniar R, Rawski G, Yang J, Johnson B (2014) Technology acceptance model (TAM) and social media usage: an empirical study on Facebook. J Enterp Inf Manag 27(1):6-30. https://doi.org/10.1108/ JEIM-04-2012-0011

Rogers EM (2003) Diffusion of innovations, 5. Aufl. Free Press, New York

Sanchez-Vives MV, Slater M (2005) From presence to consciousness through virtual reality. Nat Rev Neurosci 6(4):332-339. https://doi.org/10.1038/nrn1651

Shim S, Lee Y (2011) Consumer's perceived risk reduction by 3D virtual model. Int J Retail Distrib Manag 39(12):945-959. https://doi.org/10.1108/09590551111183326

Sjurts I (2011) Gabler Lexikon Medienwirtschaft, 2. Aufl. Springer, Wiesbaden

Steuer J (1992) Defining virtual reality: dimensions determining telepresence. J Commun Manag 42(4):73-93. https://doi.org/10.1111/j.1460-2466.1992.tb00812.x

Stone M (1974) Cross-validatory choice and assessment of statistical predictions. J R Stat Soc Series B Stat Methodol 36(2):111-147

Taylor JW (1974) The role of risk in consumer behavior. J Mark 38(2):54-60. https://doi.org/10.2307/ 1250198

Tseng SY, Wang CN (2016) Perceived risk influence on dual-route information adoption processes on travel websites. J Bus Res 69(6):2289-2296. https://doi.org/10.1016/j.jbusres.2015.12.044

Venkatesh V (2000) Determinants of perceived ease of use: integrating control, intrinsic motivation, and emotion into the technology acceptance model. Inf Syst Res 11(4):342-365. https://doi.org/10.1287/ isre.11.4.342.11872

Venkatesh V, Morris MG, Davis GB, Davis FD (2003) User acceptance of information technology: toward a unified view. Mis Q 27(3):425-478. https://doi.org/10.2307/30036540

Vessey I (1991) Cognitive fit: a theory-based analysis of the graphs versus tables literature. Decis Sci 22(2):219-240

Wu LL, Lee L, Su HY (2008) Consumers' acceptance of Websites for pre-purchase information seeking. J Inf Manag 15(3):227-250

Xiang Z, Magnini VP, Fesenmaier DR (2015) Information technology and consumer behavior in travel and tourism: Insights from travel planning using the internet. J Retail Consum Serv 22:244-249. https:// doi.org/10.1016/j.jretconser.2014.08.005

Zipf GK (2012) Human behavior and the principle of least effort: An introduction to human ecology. Martino Publishing, Mansfield Centre 